\title{
Encefalopatía posterior reversible como primera manifestación del Síndrome de Guillain-Barré
}

\author{
SERGIO URRUTIA L. ${ }^{1}$, EDUARDO VENEGAS P. ${ }^{1}$, \\ CRISTIÁN FIGUEROA V. ${ }^{1}$, CATALINA CARRIZO C. ${ }^{2}$
}

'Unidad de Neurología Adultos, Hospital Clínico FUSAT, Rancagua, Chile. ${ }^{2}$ Centro de Salud Familiar, Rancagua, Chile.

Sin fuente de apoyo financiero

Recibido el 14 de marzo de 2011, aceptado el 17 de mayo de 2012

Correspondencia a: Dr. Sergio Urrutia Lagos. E-mail:drsergiourrutia@ gmail.com

\section{Posterior reversible encephalopathy as the first manifestation of Guillain-Barré syndrome. Report of one case}

We report a 56 year old male hypertensive, who presented with a posterior reversible encephalopathy syndrome (PRES) as an initial manifestation of Guillain-Barré syndrome (GBS). His first symptoms were right hemiparesis and hemihypoesthesia, followed by headache, dizziness, dysarthria and a general feeling of discomfort. On the third day, flaccid tetraparesis, impairment of consciousness, epileptic seizures and respiratory failure appeared, along with severe hypertension. Cerebral Magnetic Resonance Imaging showed the characteristic PRES lesions. Cerebrospinal fluid analyses revealed albumin-cytological dissociation and nerve conduction studies showed an axonal demyelinating polyradiculoneuropathy, which confirmed the diagnosis of GBS. Treatment with intravenous immunoglobulin was given together with antihypertensive therapy and mechanical ventilation, achieving an important clinical and imaging remission of PRES, but maintaining tetraparesis during the hospitalization. Twelve months after discharge and regular motor rehabilitation, the patient achieved complete autonomy on the activities of daily living. It has been postulated that the autonomic failure and the elevation of circulating pro-inflammatory cytokines in GBS may be the cause of a breach in the blood-brain barrier, thus causing PRES, that can completely remit with an adequate management.

(Rev Med Chile 2012; 140: 1316-1320).

Key words: Guillain-Barré syndrome; Hypertensive encephalopathy; Posterior leukoencephalopathy syndrome.
E 1 síndrome de encefalopatía posterior reversible (SEPR), también denominado encefalopatía por hiperperfusión, comúnmente se manifiesta con cefalea, vómitos, trastorno de conciencia, alteraciones visuales, crisis epilépticas y paresia de extremidades. Descrito por primera vez en $1996^{1}$, es una entidad clínico-radiológica poco frecuente, que se debe a una pérdida de la autorregulación circulatoria cerebral asociado a una disfunción endotelial, generalmente secundaria a una crisis de hipertensión arterial (HTA). También se ha relacionado con eclampsia, trasplante de órganos, enfermedades autoinmunes, falla renal aguda, terapia inmunosupresora e inmunoglobulina intravenosa (IgIV), entre otras ${ }^{2}$. La resonancia nuclear magnética (RNM) muestra lesiones preferentemente en regiones cerebrales posteriores, hiperintensas en secuencias T2 e isointensas o hipointensas en imágenes ponderadas en difusión, con aumento del coeficiente de difusión aparente, hallazgos compatibles con edema vasogénico ${ }^{3}$. La corrección oportuna del factor causal produce la regresión total de los síntomas y de las lesiones cerebrales, salvo cuando existe edema citotóxico capaz de producir un daño irreversible.

Por otra parte, el síndrome de Guillain-Barré 
(SGB) puede cursar con complicaciones autonómicas como HTA, taquicardia sinusal, hipotensión postural y cambios electrocardiográficos. También pueden ocurrir otras complicaciones graves como taquiarritmias o bradiarritmias con paro cardiaco ${ }^{4}$.

Existen pocos reportes de casos que muestren una asociación entre SEPR y SGB. A continuación presentamos un paciente que desarrolló ambos cuadros clínicos.

\section{Caso clínico}

Hombre de 56 años de edad con antecedente de HTA bien controlada en monoterapia, inició su cuadro clínico con pérdida de fuerza en su mano derecha y dificultad para diferenciar los cambios de temperatura del agua de la llave. En pocas horas el déficit motor progresó hacia una paresia facio-braquio-crural derecha leve, que no le impidió continuar con sus actividades habituales. Al día siguiente se agregó cefalea, mareos, disartria y compromiso del estado general. A partir del tercer día refirió debilidad muscular de ambas piernas, que evolucionó en forma progresiva y ascendente, afectando sus cuatro extremidades e impidiendo la marcha al sexto día de evolución. En esta primera semana controló diariamente su presión arterial, documentando cifras cercanas a los 200/100 mmHg. Consultó en su hospital local, donde presentó compromiso cuantitativo de conciencia y una crisis epiléptica versiva. Debido a este empeoramiento clínico fue trasladado a nuestra institución cursando una falla respiratoria.

$\mathrm{Al}$ ingreso en la Unidad de Cuidados Intensivos y conectado a ventilación mecánica, se registró presión arterial 220/90 $\mathrm{mmHg}$, frecuencia cardiaca $80 \mathrm{x}$ min, temperatura axilar $37^{\circ} \mathrm{C}$ y glicemia capilar $110 \mathrm{mg} / \mathrm{dl}$. El examen físico general no mostró hallazgos patológicos. El examen neurológico reveló una tetraparesia fláccida, arreflexia osteotendínea global, reflejos plantares flexores, respuesta al dolor simétrica y reflejos troncoencefálicos preservados. Destacó en la bioquímica plasmática una creatinkinasa total (CKt) de 677, con función renal, función hepática y electrolitos plasmáticos en rangos normales. La RNM de cerebro en secuencia FLAIR mostró lesiones hiperintensas bilaterales sobre regiones parieto-occipitales y frontales, sugerentes de edema vasogénico (Figura 1A). El estudio del líquido cefalorraquídeo (LCR) mostró proteínas elevadas en $169 \mathrm{mg} / \mathrm{dl}$, con citología y glucosa normales. Posteriormente, la neuroconducción (NC) de cuatro extremidades evidenció ausencia de ondas $\mathrm{F}$ y de conducción sensitiva global, potenciales motores compuestos de baja amplitud y velocidades muy disminuidas,
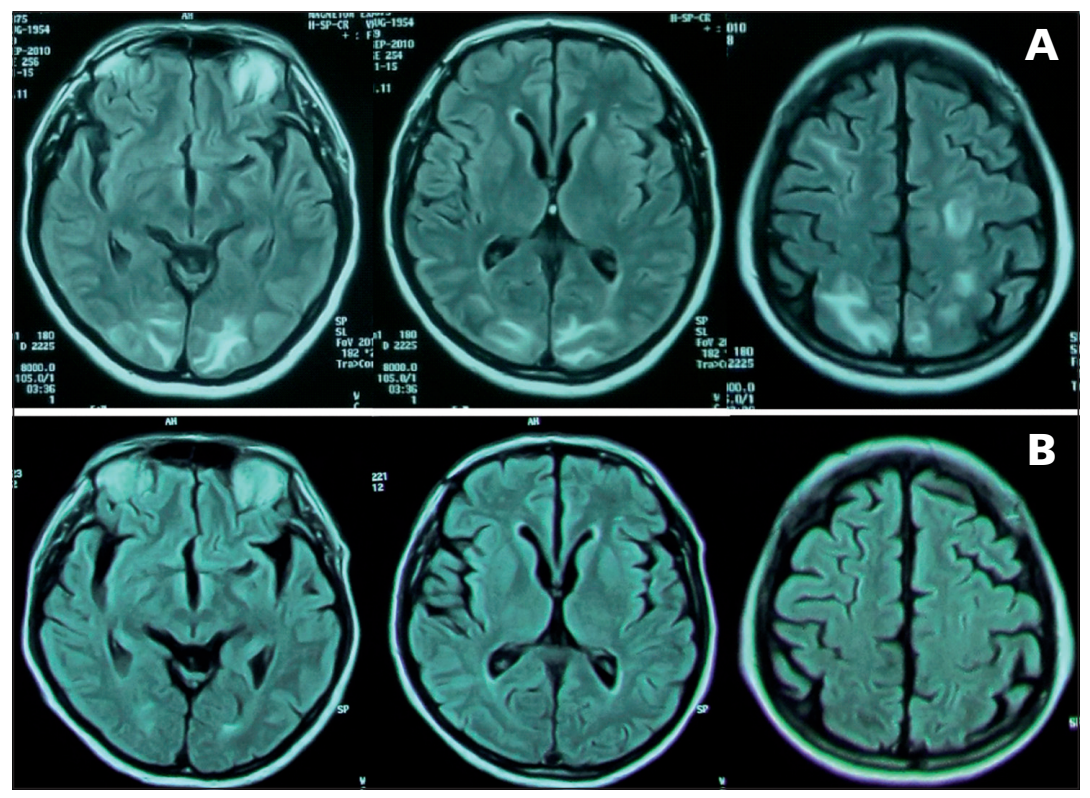

Figura 1. RNM de cerebro, cortes axiales en secuencia FLAIR: (A) Lesiones hiperintensas de predominio subcortical en regiones parieto-occipitales y frontales, compatibles con edema vasogénico, hallazgo característico del SEPR (B) Resolución total de las lesiones cinco meses después. 
con algunos elementos de bloqueo de conducción nerviosa (Figura 2), hallazgos que son compatibles con una polirradiculoneuropatía desmielinizante y axonal severa.

El tratamiento del SGB se realizó con IgIV en dosis de $0,4 \mathrm{~g} / \mathrm{kg} /$ día durante 5 días. Como terapia antihipertensiva se utilizó labetalol endovenoso en infusión continua, con el objetivo de lograr cifras menores a $140 / 90 \mathrm{mmHg}$, en un descenso paulatino no mayor al 20\% diario, meta lograda al finalizar la primera semana.

Durante la etapa aguda de la enfermedad, su evolución global fue satisfactoria, otorgándose el alta luego de 40 días de hospitalización. El examen neurológico de ese momento mostraba una tetraparesia de predominio distal, con plejía de pies y manos.

Luego de 12 meses de rehabilitación ambulatoria, su déficit motor mejoró satisfactoriamente, logrando la independencia total en sus actividades de la vida diaria. El examen neurológico detallado sólo exhibió una discreta paresia de la flexo-extensión de ortejos y pies, con una leve hiperreflexia osteotendínea braquiocrural derecha. La RNM cerebral de control mostró una regresión completa de las lesiones antes señaladas (Figura 1B). Por otra parte, mantiene nuevamente un buen control de la HTA con su fármaco habitual.

\section{Discusión}

Nuestro paciente reúne las características principales de un $\mathrm{SEPR}^{1} \mathrm{y}$ de un $\mathrm{SGB}^{5}$. Por una parte, el desarrollo de alteraciones sensitivo-motoras de un hemicuerpo, seguido por cefalea, mareos, decaimiento, disartria, trastorno de conciencia y crisis epiléptica versiva, hicieron pensar en un compromiso encefálico. Estos síntomas, asociados al registro de crisis hipertensivas y a los hallazgos característicos en la RNM cerebral, permitieron hacer el diagnóstico definitivo de un SEPR. La expresión de síntomas focales en nuestro paciente, sería explicada por la distribución asimétrica de las lesiones vistas en la RNM cerebral, especialmente a nivel de los lóbulos frontales. Otros síntomas globales, como el compromiso de conciencia, estarían determinados no sólo por la presencia de edema vasogénico bihemisférico, si no también por el trastorno metabólico cerebral difuso observado en esta patología, independiente de la carga lesional en la $\mathrm{RNM}^{10}$.

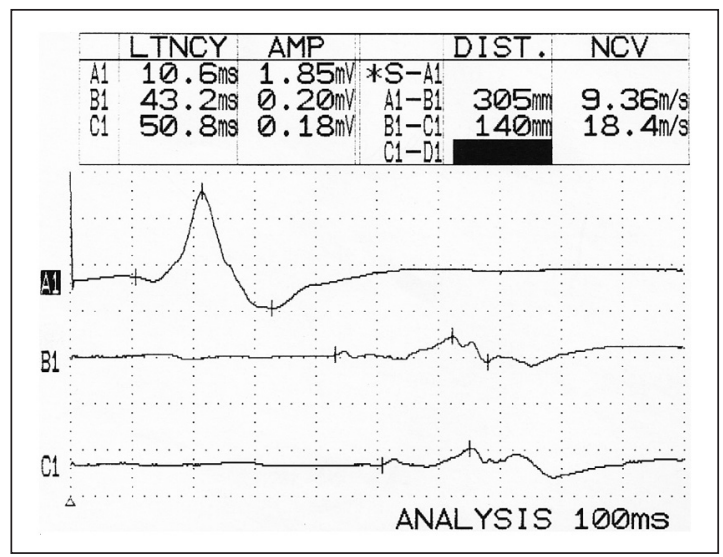

Figura 2. Conducción motora del nervio cubital izquierdo mostrando latencia distal prolongada, baja amplitud de la respuesta $\mathrm{M}$, reducción de la velocidad de conducción, con dispersión y caída de la amplitud del potencial motor compuesto en las estimulaciones proximales, compatibles con fenómenos de bloqueo de conducción.

Por otra parte, la aparición de una debilidad muscular ascendente que en pocos días evolucionó a una tetraparesia fláccida arrefléctica, con compromiso de músculos ventilatorios e indemnidad de troncoencéfalo, fundamentaron el diagnóstico clínico de un SGB y el inicio del tratamiento con IgIV. El LCR con disociación albúmino-citológica y posteriormente la neuroconducción con hallazgos característicos, respaldaron el diagnóstico ${ }^{6}$. La elevación de CKt en rango leve, es un hallazgo habitual en esta patología?

Desde la descripción inicial del SEPR, realizada por Hinchey en el año 1996', se han propuesto como mecanismos patogénicos la pérdida en la autorregulación circulatoria cerebral y la disfunción endotelial. En la mayoría de los casos, ocurre una crisis hipertensiva que sobrepasa los mecanismos de autorregulación, provocando un estado de hiperperfusión cerebral que conlleva al quiebre de la barrera hematoencefálica, dando como resultado la salida forzada de elementos intravasculares hacia el parénquima cerebral, lo que se conoce como edema hidrostático, un tipo específico de edema vasogénico. A su vez, en los casos donde no hay crisis hipertensiva, se sugiere que son toxinas circulantes las que provocan un daño endotelial directo y el consecuente edema vasogénico ${ }^{1,8}$.

Es llamativo observar, que las lesiones ocasionadas en el SEPR ocurren principalmente sobre 
regiones cerebrales posteriores, lo que probablemente se deba a la menor inervación simpática adrenérgica encontrada en las arterias de circulación posterior, lo cual las haría más vulnerables a las alteraciones antes mencionadas?.

En la mayoría de los casos de SGB, es posible detectar algún grado de compromiso autonómico, así el $61 \%$ de los pacientes desarrolla una HTA transitoria entre los días 2 y 21 de su evolución ${ }^{10}$. A través de este mecanismo, podría ocurrir una hiperperfusión cerebral capaz de producir el quiebre en la barrera hematoencefálica y así ocasionar un SEPR. Se ha logrado encontrar niveles elevados de catecolaminas urinarias en el momento de mayor compromiso motor del $\mathrm{SGB}^{11,12}$, lo que apoyaría esta base etiopatogénica. Además, es posible encontrar en el SGB un exceso de citoquinas proinflamatorias circulantes ${ }^{13}$, las que favorecerían también la pérdida de la autorregulación circulatoria cerebral ${ }^{14}$.

A pesar de la relación existente, son escasos los pacientes que desarrollan un SEPR como complicación de un SGB. Algunas publicaciones muestran pacientes que debutaron con un SGB, y que luego de ser tratados con IgIV desarrollaron el SEPR ${ }^{15,16}$, atribuyendo a este fármaco un factor de riesgo para desarrollar HTA. Otras, por el contrario, muestran pacientes que cursaron con el SEPR antes de recibir cualquier tratamiento dirigido al SGB ${ }^{11,17-20}$, lo que sugirió la existencia de un vínculo directo entre ambas patologías independiente del uso de fármacos. En este grupo encontramos quienes desarrollaron el SEPR previo a las manifestaciones clásicas del $\mathrm{SGB}^{17-19}$, teniendo en común debutar con una encefalopatía, registrar HTA severa al inicio de los síntomas, tener lesiones típicas de un SEPR en la RNM, desarrollar un SBG a los pocos días de evolución y lograr una regresión completa de ambos cuadros luego de ser tratados con IgIV y fármacos antihipertensivos. Nuestro paciente comparte con ellos, similares aspectos clínico-radiológicos y una excelente respuesta a tratamiento.

El hecho de desarrollar una elevación severa y sostenida de las cifras tensionales, siendo un paciente que mantiene un control crónico satisfactorio de su HTA con monoterapia, y que una vez tratados el SEPR y el SGB logra retomar con éxito su tratamiento basal, nos hace postular que el factor gatillante de este descontrol fue el compromiso autonómico del SGB.
Esta comunicación estimula a mantenerse alerta a las complicaciones neurológicas poco frecuentes de la HTA, además a no olvidar que el compromiso autonómico de un SGB puede ser de tal magnitud como para desencadenar un SEPR, cuadro grave y potencialmente letal, pero capaz de revertir en forma completa con un manejo oportuno.

\section{Referencias}

1. Hinchey J, Chaves C, Appignani B, Breen J, Pao L, Wang A, et al. A reversible posterior leukoencephalopathy syndrome. N Engl J Med 1996; 334: 494-500.

2. Bartynski WS. Posterior Reversible Encephalopathy Syndrome, Part 1: Fundamental Imaging and Clinical Features. Am J Neuroradiol 2008; 29: 1036-42.

3. Lamy C, Oppenheim C, Meder JF, Mas JL. Neuroimaging in posterior reversible encephalopathy syndrome. J Neuroimaging 2004; 14: 89-96.

4. Flachenecker P, Wermuth P, Hartung HP, Reiners K. Quantitative assessment of cardiovascular autonomic function in Guillain-Barré syndrome. Ann Neurol 1997; 42: 171-9.

5. Van Doorn PA, Ruts L, Jacobs BC. Clinical features, pathogenesis, and treatment of Guillain-Barré syndrome. Lancet Neurol 2008; 7: 939-50.

6. Uncini A, Yuki N. Electrophysiologic and immunopathologic correlates in Guillain-Barré syndrome subtypes. Expert Rev. Neurother 2009; 9 (6): 869-994.

7. Ropper A, Shahani B. Pain in Guillain Barré Syndrome. Arch Neurol 1984; 41: 511-4.

8. Covarrubias D, Luetmer P, Campeau N. Posterior Reversible Encephalopathy Syndrome: Prognostic Utility of Quantitative Diffusion-Weighted MR Images. AJNR Am J Neuroradiol 2002; 23: 1038-48.

9. Edvinsson L, Owman C, Sjöberg NO. Autonomic nerves, mast cells, and amine receptors in human brain vessels. A histochemical and pharmacological study. Brain Res. 1976; 15 (3): 377-93.

10. Eichler FS, Wang P, Wityk RJ, Beauchamp NJ, Barker PB. Diffuse Metabolic Abnormalities in Reversible Posterior Leukoencephalopathy Syndrome. Am J Neuroradiol. 2002; 23: 833-7.

11. Louie J, Igbokwe E, Hinchey J. Posterior Reversible Encephalopathy Associated with the Dysautonomia of Guillain-Barré Syndrome. Neurological Bulletin 2009; 1: 7-10.

12. Lebrun C, Ghetau G, Candito M, Chatel M. [Acute polyradiculoneuritis and dysautonomia: contribution 
of assaying catecholamines and their methoxylated metabolites]. Rev Neurol (Paris) 2001; 157: 304-8.

13. Kieseier BC, Tani M, Mahad D, Oka N, Ho T, Woodroofe $\mathrm{N}$, et al. Chemokines and chemokine receptors in inflammatory demyelinating neuropathies: a central role for IP-10. Brain 2002; 125: 823-34.

14. Wong D, Dorovini-Zis K, Vincent SR. Cytokines, nitric oxide, and cGMP modulate the permeability of an in vitro model of the human blood-brain barrier. Exp Neurol 2004; 190: 446-55.

15. Mathy I, Gille M, Van Raemdonck F, Delbecq J, Depre A. Neurological complications of intravenous immunoglobulin (IVIg) therapy: an illustrative case of acute encephalopathy following IVIg therapy and a review of the literature. Acta Neurol Belg 1998; 98: 347-51.

16. Doss-Esper CE, Singhal AB, Smith MS, Henderson GV. Reversible posterior leukoencephalopathy, cerebral vasoconstriction, and strokes after intravenous immune globulin therapy in Guillain-Barré syndrome. J Neuroimaging 2005; 15: 188-92.

17. Elahi A, Kelkar P, St Louis EK. Posterior reversible encephalopathy syndrome as the initial manifestation of Guillain-Barré syndrome. Neurocrit Care 2004; 1: 465-8.

18. Van Diest D, Van Goethem JW, Vercruyssen A, Jadoul C, Cras P. Posterior reversible encephalopathy and Guillain-Barré syndrome in a single patient: coincidence or causative relation? Clin Neurol Neurosurg 2007; 109: 58-62.

19. Sutter R, Mengiardi B, Lyrer P, Czaplinski A. Posterior reversible encephalopathy as the initial manifestation of a Guillain-Barré syndrome. Neuromuscular Disorders 2009; 19: 709-10.

20. Bavikatte G, Gaber T, Eshiett MU-A. Posterior reversible encephalopathy syndrome as a complication of GuillainBarré syndrome. Journal of Clinical Neuroscience 2010; 17: 924-6. 